\title{
The Effects of Excitotoxic Hippocampal Lesions in Rats on Risperidone- and Olanzapine- Induced Locomotor Suppression
}

\author{
Mark E. Bardgett, William M. Humphrey, and John G. Csernansky
}

\begin{abstract}
Previous studies have shown that excitotoxic hippocampal lesions in rats attenuate the ability of different doses of haloperidol, but not of clozapine, to suppress locomotor activity. The purpose of the present study was to determine if kainic acid-induced hippocampal damage reduces the degree of locomotor suppression produced by two relatively newer antipsychotic drugs, risperidone and olanzapine. Young adult male rats received bilateral intracerebroventricular infusions of the excitotoxin, kainic acid (KA), or vehicle and were tested for locomotor responses to drug treatment 30 days later. Infusions of $K A$ produced neuronal loss in the CA3 region of the dorsal hippocampus in every rat. As reported previously, KA lesions reduced the ability of haloperidol $(0.35 \mathrm{mg} / \mathrm{kg})$ to completely suppress the locomotor activity elicited by amphetamine $(1.5 \mathrm{mg} / \mathrm{kg})$ relative to the effect of haloperidol
\end{abstract}

in non-lesioned controls. Lesioned animals treated with a moderate dose of risperidone $(1.4 \mathrm{mg} / \mathrm{kg})$ also exhibited significantly more locomotor activity after amphetamine treatment in comparison to control animals. A trend toward greater activity was also observed in the lesioned group relative to the control group after olanzapine $(3.0 \mathrm{mg} / \mathrm{kg}$ ) injection ( $\mathrm{p}=.09,2$-tailed). The locomotor effects of lower and higher doses of risperidone and olanzapine were not altered by kainic acid lesions. These data suggest that the locomotor-suppressive effects of moderate doses of risperidone and, perhaps, olanzapine involve hippocampal neurons, but that higher doses of each drug can suppress activity in a hippocampal-independent manner.

[Neuropsychopharmacology 27:930-938, 2002] (C) 2002 American College of Neuropsychopharmacology. Published by Elsevier Science Inc.
KEY WORDS: Antipsychotic; Hippocampus; Amphetamine; Haloperidol; Locomotion; Grooming

Many neuroimaging studies have revealed reduced volume or related alterations of the hippocampus and related limbic-cortical structures in some people with

From the Department of Psychiatry, Washington University School of Medicine, St. Louis, MO

Address correspondence to: Dr. M.E. Bardgett, Department of Psychology, Northern Kentucky University, Nunn Drive, Highland Heights, KY 41099. Tel.: (859) 572-5591; Fax: (859) 572-6085; E-mail: bardgettm@nku.edu

Received January 29, 2002; revised May 14, 2002; accepted May 20, 2002.

Online publication: 5/22/02 at www.acnp.org/citations/ Npp052202310/default.htm. schizophrenia (for review, see McCarley et al. 1999) and Alzheimer's disease (e.g., Csernansky et al. 2000). Neurons within the hippocampus and other limbic-cortical structures project to the nucleus accumbens shell, a primary locus of antipsychotic drug action (Deutch 1996). Since brain regions involved in antipsychotic drug action receive input from limbic-cortical structures, it is plausible that limbic-cortical neurons modulate antipsychotic drug action. One way to determine if limbiccortical neurons contribute to antipsychotic drug action is to assess the effects of limbic-cortical lesions in animals on locomotor responses to antipsychotic drugs. The ability of drug compounds to reduce spontaneous locomotor activity and amphetamine-induced elevations in locomotor activity has served as a principal preclinical 
assay of antipsychotic drug action (Arnt 1995; Ogren 1996). However, despite the evidence for hippocampal deficits in disorders treated with antipsychotics, very few studies have considered the possibility that hippocampal lesions can alter the ability of antipsychotic drugs to suppress both forms of locomotor activity (Bardgett and Csernansky 1996).

Our research has characterized the impact of limbiccortical deficits on antipsychotic drug action by studying animals with limbic-cortical neuropathology induced by the intracerebroventricular (ICV) administration of kainic acid (KA). Animals treated with KA exhibit neuronal loss predominantly in the dorsal CA3 pyramidal cell field of the hippocampus. A minority of the animals also display cell loss and/or gliosis in the CA1 pyramidal cell field of the hippocampus, in the laterodorsal thalamus, the amygdala, and/or the posterior piriform cortex. We have previously demonstrated that KA lesions can alter behavioral and biochemical responses to some antipsychotic drugs. For instance, haloperidol, a typical antipsychotic drug, has a reduced capacity to decrease spontaneous and amphetamine-elevated locomotor activity in KA-lesioned rats. However, clozapine, an atypical antipsychotic drug, retains its capacity to suppress both forms of locomotor activity in KA-lesioned animals (Bardgett et al. 1998). Consistent with these observations, the ability of haloperidol to elevate dopamine turnover is reduced in the nucleus accumbens of KA-lesioned animals, while the ability of clozapine to increase dopamine turnover in the same brain region is normal (Bardgett et al. 1997b).

Risperidone and olanzapine are presently the most widely used antipsychotic drugs, and their atypical properties in patients have been asserted (Marder 1999; Tollefson and Kuntz 1999). The purpose of this study was to determine if KA lesions alter the locomotor suppressive effects of risperidone and olanzapine on spontaneous and amphetamine-elevated locomotor activity. To test the effects of KA lesions on locomotor responses to risperidone, spontaneous and amphetamine-elevated locomotor activity were recorded in lesioned and control animals after acute injections of haloperidol, different doses of risperidone, or saline. The effects of KA lesions on locomotor responses to olanzapine were tested in a second experiment in a similar manner.

\section{METHODS}

\section{Animals}

All experimental procedures were performed according to the Current Guide for the Care and Use of Laboratory Animals (USPHS) under a protocol approved by the Washington University Animal Studies Committee. Seventy-day-old male Sprague-Dawley rats (Harlan Bioproducts, Indianapolis, IN) were used in these ex- periments. A total of 11-12 KA-lesioned rats and 18 control rats were used to test locomotor responses to risperidone. A total of 11-12 KA-lesioned rats and 15 control rats were used to test responses to olanzapine. (A lesioned animal in each experiment died due to unknown causes.)

\section{Kainic Acid Lesions}

Prior to ICV administration of KA, animals were anesthetized with sodium pentobarbital (Sigma, $50.0 \mathrm{mg} / \mathrm{kg}$ IP). Supplemental doses of sodium pentobarbital (21.0 $\mathrm{mg} / \mathrm{kg}$ IP) were administered as needed. Each rat was placed in a Kopf stereotaxic frame, and the skin above the skull was shaved and swabbed with Betadine. A 2 $\mathrm{cm}$ incision was made in the skin parallel to the midline above the lateral ventricles and a small hole was made in the skull above each lateral ventricle (AP -1.2, $\mathrm{ML} \pm$ 1.4, V -4.4 relative to bregma; Paxinos and Watson 1986). A 25-gauge needle, attached to PE-10 tubing and a 10 microliter Hamilton syringe, was lowered into the right lateral ventricle over a 1-min period. One microliter of KA $(0.8 \mathrm{mg} / \mathrm{ml})$ was infused into one ventricle at a time over a $20 \mathrm{~min}$ period. This time period was selected to minimize collateral neuronal damage, based on previous work from our laboratory (Bardgett et al. 1995, 1997a) and others (Nadler et al. 1978). Kainic acid (Sigma) was dissolved in artificial cerebrospinal fluid (ACSF) containing $2.7 \mathrm{mM} \mathrm{KCl}, 145 \mathrm{mM} \mathrm{NaCl}, 1.0 \mathrm{mM}$ $\mathrm{MgCl}_{2}, 1.2 \mathrm{mM} \mathrm{CaCl}, 2.0 \mathrm{mM} \mathrm{Na} \mathrm{HPO}_{4}$, pH 7.4. All chemicals used in the ACSF were obtained from Sigma. Infusions were controlled by a Harvard infusion pump (Pump 22, Harvard Instruments, Cambridge, MA). The needle was left in place for $2 \mathrm{~min}$ after infusion. The needle was then removed from the right ventricle, and the procedure was repeated for the left ventricle. Control animals received similar infusions of ACSF only. Animals that received KA exhibited vibrissa twitching and/or clonic movements of the forelimbs for several hours post-infusion. Most animals were given a supplemental dose of sodium pentobarbital after surgery in addition to $3 \mathrm{cc}$ (SC) of $0.9 \%$ saline containing 5\% sucrose. Animals did not exhibit seizure activity at $24 \mathrm{~h}$ post-surgery or at any time thereafter.

\section{Drugs}

In the assessment of locomotor responses to antipsychotic drugs, each animal received an injection of saline or an antipsychotic drug, and $30 \mathrm{~min}$ later, locomotor activity was recorded before and after an injection of Damphetamine sulfate (Sigma, $1.50 \mathrm{mg} / \mathrm{kg} \mathrm{SC}$ ). This dose of amphetamine was dissolved in saline and has been found in previous studies to produce a reliable increase in locomotion (Bardgett et al. 1997a, 1998; Bardgett and Henry 1999). Animals in the risperidone group received 


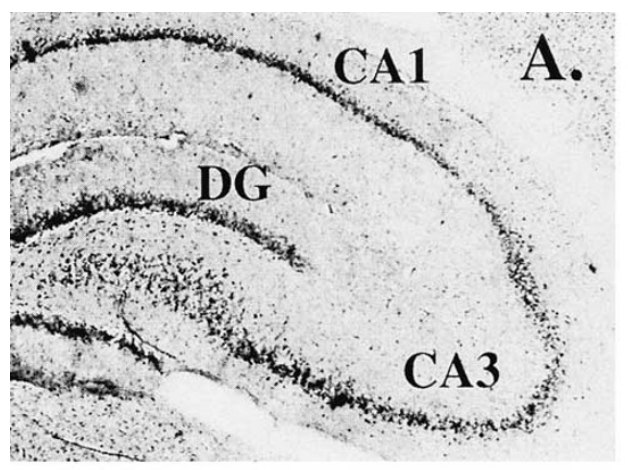

CONTROL

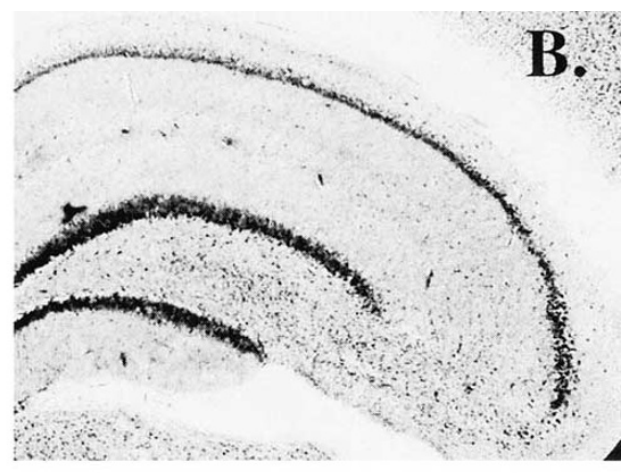

KAINIC ACID LESION
Figure 1. Photomicrographs of the dorsal hippocampus from a control animal (A) and an animal treated with ICV KA (B). Note that in the section from a KA-treated animal, the dentate gyrus (DG) and CA1 region is well-preserved, while the CA3 region has extensive cell loss. injections of either vehicle or $0.14,1.4$, and $5.0 \mathrm{mg} / \mathrm{kg}$ risperidone (SC) in a counterbalanced order. Animals in the olanzapine group received injections of vehicle or $1.0,3.0$, and $10.0 \mathrm{mg} / \mathrm{kg}$ olanzapine (SC) in a counterbalanced order. The doses of risperidone and olanzapine were selected to encompass the $\mathrm{ED}_{50}$ of each drug for reducing locomotor activity elicited by amphetamine (Arnt 1995). For comparison, locomotor responses to a single dose of haloperidol $(0.35 \mathrm{mg} / \mathrm{kg}, \mathrm{SC})$ were also studied in each group of lesioned and control animals. This dose of haloperidol was based on our previously published data (Bardgett et al. 1998), which demonstrated that lesioned animals were less sensitive than controls to its locomotor-suppressing effects. Animals were tested with one drug dose per week. All antipsychotic drugs were dissolved in $\mathrm{HCl}$ or glacial acetic acid (olanzapine) and brought to an appropriate volume with $0.9 \%$ saline and a $\mathrm{pH}$ of approximately 6.0 with $\mathrm{NaOH}$.

\section{Assessment of Locomotor Activity and Grooming}

Assessment of locomotor activity after antipsychotic drug injection was initiated 30 days after surgery. On testing days, each animal received an injection of risperidone, olanzapine, haloperidol, or saline and was placed back in its home cage. Thirty minutes later, animals were placed in clear Plexiglas testing cages $(51 \mathrm{~cm}$ long $\times 26.5 \mathrm{~cm}$ wide $\times 32 \mathrm{~cm}$ high). Testing was conducted in a darkened room illuminated by a $25 \mathrm{~W}$ lamp. Each cage was equipped with two photoelectric movement sensors (Comet 100 Series Reflex sensors, Eaton Corp., Everett, WA) that projected beams of visible red light across the width of the testing cage at 17 and 34 $\mathrm{cm}$ along the length of the cage, and at a height of $7 \mathrm{~cm}$ from the bottom of the cage. Locomotor activity was recorded for $30 \mathrm{~min}$, and then animals were removed from the testing cage and received an injection of amphetamine. Following amphetamine injection, animals were immediately returned to the testing cages, and locomotor activity was recorded for an additional $60 \mathrm{~min}$.
Stereotypic grooming behavior (self-directed sniffing, licking, biting, or scratching with forelimbs or hindlimbs) was also assessed for $15 \mathrm{~s}$ every $20 \mathrm{~min}$ after amphetamine injection as a indirect measure of nigrostriatal dopamine function (Prosser et al. 1989; Mittleman et al. 1991, 1993). Scores were rated as described in Bardgett et al. (1997a): 0 - no grooming, 1 - grooming for less than $3 \mathrm{~s}, 2$ - grooming for more than $3 \mathrm{~s}, 4$ - continuous grooming. The scores were averaged for the three time samples.

\section{Histology}

At the completion of the behavioral assessments, each animal was sacrificed by pentobarbital overdose, and the brain removed and frozen. Frozen coronal sections $(40 \mu \mathrm{m})$ of each brain were cut on a cryostat and stained with thionin. Sections containing the dorsal hippocampus, the anterior thalamus, the basolateral amygdala, the piriform cortex, the ventral hippocampus, and the entorhinal cortex were inspected for gross pathology (i.e. cell loss, morphological changes, gliosis) as previously described (Bardgett et al. 1997a).

\section{Data Analysis}

For each drug (risperidone or olanzapine), the effects of KA lesions on spontaneous locomotor activity and amphetamine-elevated locomotor activity were analyzed separately. Spontaneous locomotor activity was defined as the total number of photobeam beam breaks generated every five minutes by each animal during the $30 \mathrm{~min}$ prior to amphetamine injection. Amphetamineelevated locomotor activity was defined as the total number of photobeam breaks generated every five minutes by each animal during the 60 min following amphetamine injection. The locomotor activity of lesioned and control animals was compared at each drug dose using a 2-way analysis of variance (ANOVA) with lesion as a between-group factor and time as a within- 
group factor. For grooming, scores were compared using a 2-way ANOVA with lesion and drug as between group factors. Post-hoc testing was performed using Fisher's protected least-squares difference (PLSD) test and significant differences were accepted for $p<.05$ (2-tailed).

\section{RESULTS}

\section{Histology}

Visual inspection of thionin-stained brain sections at the light microscopic level showed that KA-treated animals $(n=22)$ sustained neuronal cell loss in the CA3 pyramidal cell field of the dorsal hippocampus (Figure 1). A minority of the animals treated with KA also exhibited cell loss and/or gliosis in the CA1 region of the dorsal hippocampus $(\mathrm{n}=10)$, the laterodorsal thalamus $(\mathrm{n}=4)$ and the posterior piriform cortex $(n=8)$. None of the animals demonstrated frank cell loss or gliosis in the amygdala. There were no obvious differences in the extent of the pathology between cohorts of animals used in the two experiments.

\section{Experiment 1: Effects of KA lesions on Locomotor and Grooming Responses to Risperidone}

In the experiment comparing locomotor responses of KA-lesioned and control animals to risperidone, both groups of animals demonstrated similar amounts of spontaneous locomotor activity after receiving saline injections alone and similar levels of increased locomotor activity after receiving SC injections of $\mathrm{d}$-amphetamine sulfate $(1.5 \mathrm{mg} / \mathrm{kg})$ (Figure 2, panel A). Haloperidol injection $(0.35 \mathrm{mg} / \mathrm{kg} \mathrm{SC})$ suppressed spontaneous locomotor activity to a similar level in both lesioned and control animals. However, as previously reported (Bardgett et al. 1998), the ability of haloperidol to suppress amphetamineelevated locomotor activity was reduced in animals with KA lesions (Group effect: $\mathrm{F}_{1,14}=6.5, p<.02$ ) (Figure 2 , panel B). Lesioned animals were significantly more active than controls at 5, 15, and 30 min post-amphetamine injection (Fisher's PLSD post-hoc tests, $p<.02-.05$ ).

The effects of risperidone on locomotor activity were found to be time-, dose-, and lesion-dependent. The lowest dose $(0.14 \mathrm{mg} / \mathrm{kg}, \mathrm{SC})$ of risperidone had no effect on spontaneous locomotor activity or on amphetamine-elicited increases in locomotor activity in either group (compare panel A and panel $\mathrm{C}$ in Figure 2). The middle dose of risperidone $(1.4 \mathrm{mg} / \mathrm{kg} \mathrm{SC})$ suppressed spontaneous locomotor activity in both lesioned and control animals (Figure 2, panel D). However, after amphetamine injection, the lesioned animals were significantly more active than controls in a time-dependent manner (Lesion $\times$ Time effect: $\mathrm{F}_{11,154}=2.5, p<.007$ ). Animals with KA lesions treated with the middle dose of risperidone displayed significantly more activity at 5 ,
10,20 , and $30 \mathrm{~min}$ post-amphetamine relative to controls (Fisher's PLSD tests, $p<.02-.04$ ). The high dose of risperidone $(5.0 \mathrm{mg} / \mathrm{kg} \mathrm{SC})$ completely suppressed spontaneous and amphetamine-elevated locomotor activity in both groups of animals (Figure 2, panel E).

There was a significant effect of haloperidol and risperidone on post-amphetamine stereotypic grooming scores (Drug effect: $\mathrm{F}_{4,69}=84.8, p<.0001$ ) (Figure 3). Haloperidol decreased grooming significantly as did the highest dose of risperidone when compared with the effects of saline on post-amphetamine grooming (Fisher's PLSD tests, $p<.0001$ for each comparison). There was a trend toward lower post-amphetamine grooming in the rats treated with $1.4 \mathrm{mg} / \mathrm{kg}$ of risperidone (Fisher's PLSD test: $p=.06$ ). The effect of lesion on post-amphetamine grooming was not statistically significant.

\section{Experiment 2: Effects of KA Lesions on Locomotor Responses to Olanzapine}

The second experiment examined the effects of olanzapine on locomotor activity in KA-lesioned and control animals. In this experiment, the effects of saline and haloperidol injections on locomotor activity (Figures 4, panels A and B) were similar to those observed in the first experiment. Animals with KA lesions displayed activity profiles that were indistinguishable from controls after saline injection alone. While haloperidol reduced activity in both groups of animals, lesioned animals were significantly more active than controls (Lesion $\times$ Time effect: $\left.F_{11,231}=1.9, p<.04\right)$. Significant between-group differences were observed at 20,25, 30, 35, 45, 55, and $60 \mathrm{~min}$ post-amphetamine injection (Figure 4, panel B).

The low dose of olanzapine $(1.0 \mathrm{mg} / \mathrm{kg}$, SC) reduced spontaneous locomotor activity to a similar extent in both groups of animals. There was a time-dependent effect of this dose of olanzapine on amphetamine-induced increases in locomotor activity (Time effect: $\left.\mathrm{F}_{11,231}=17.4, p<.0001\right)$ with greater activity levels observed in the later stages of the testing period (Figure 4, panel C). The moderate dose of olanzapine $(3.0 \mathrm{mg} / \mathrm{kg})$ also similarly reduced spontaneous locomotor activity in both groups of animals. However, there was a statistical trend toward a lesion effect on amphetamine-elevated locomotor activity after treatment with this dose (Lesion effect: $\mathrm{F}_{1,21}=3.1, p=.09$ (2-tailed)). At each post-amphetamine injection time point, the lesioned group displayed a greater mean activity level than did the control group (Figure 4, panel D). After treatment with the high dose of olanzapine $(10.0 \mathrm{mg} / \mathrm{kg})$, both lesioned and control animals exhibited similar reductions in spontaneous and amphetamine-elevated locomotor activity (Figure 4, panel E).

Haloperidol and olanzapine had divergent effects on post-amphetamine stereotypic grooming (Drug effect: 


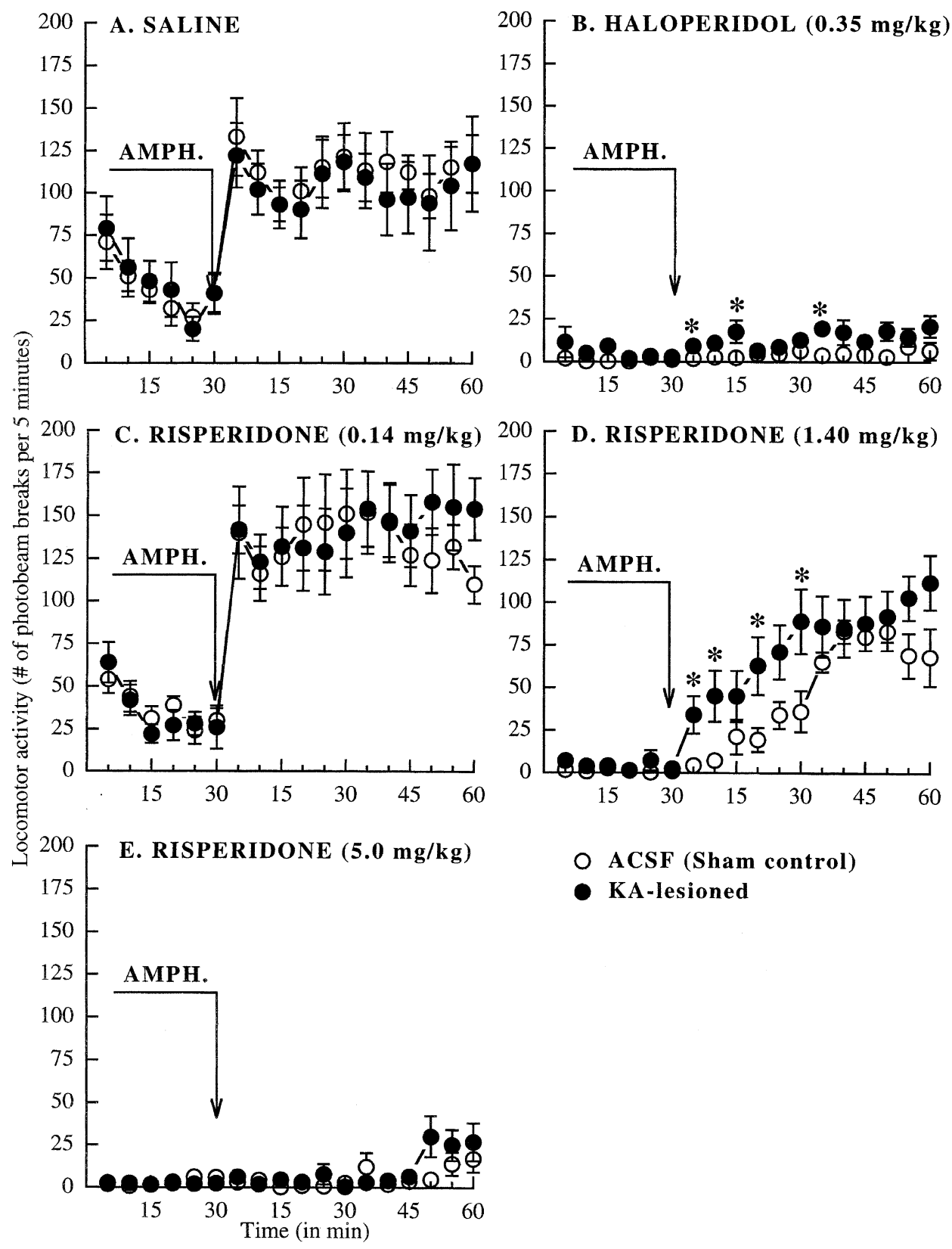

Figure 2. The effects of haloperidol and risperidone on spontaneous and amphetamineelevated locomotor activity in KA-lesioned and control rats. The drug listed at the top of each graph was injected into each animal $30 \mathrm{~min}$ prior to the recording of locomotor activity. Each circle represents the mean ( \pm SEM) number of photobeam breaks recorded from each group during 5-min bins. Spontaneous locomotor activity was recorded for the first $30 \mathrm{~min}$. After this period, D-amphetamine $(1.5 \mathrm{mg} / \mathrm{kg}$, SC) was injected into each animal, and locomotor activity was recorded for another $60 \mathrm{~min}$. Asterisks denote statistically significant differences between groups at individual time points $(p<.05$, Fisher's PLSD).

$\mathrm{F}_{4,106}=27.5, p<.0001$ ) (Figure 5). Haloperidol significantly depressed grooming in comparison to the effects of saline pretreatment (Fisher's PLSD test, $p<.0001$ ). However, the 1.0 and $3.0 \mathrm{mg} / \mathrm{kg}$ doses of olanzapine significantly increased grooming in relation to saline pretreatment (Fisher's PLSD tests: $p<.0001$ and .02 respectively), while the highest dose did not have a significant effect. There was no overall lesion effect on grooming scores.

\section{DISCUSSION}

The data from the present study suggest that hippocampal neurons play a modest yet statistically significant role in locomotor responses to antipsychotic drugs. This involvement of the hippocampus depends upon the type and the dose of the drug. As shown previously (Bardgett et al. 1998) and replicated in the present study, kainic acid-induced hippocampal damage attenuates haloperidol's ability to completely suppress the locomotor activity observed after amphetamine treatment. Most importantly, the present study extends the consideration of antipsychotic drug action in the context of hippocampal deficits to two of the most widely prescribed antipsychotics, risperidone and olanzapine. It was found that hippocampal damage attenuates the ability of a moderate dose of risperidone to reduce the locomotor activity produced by amphetamine. A somewhat similar lesion effect was observed on the response to a moderate dose of olanzapine; however this effect was 


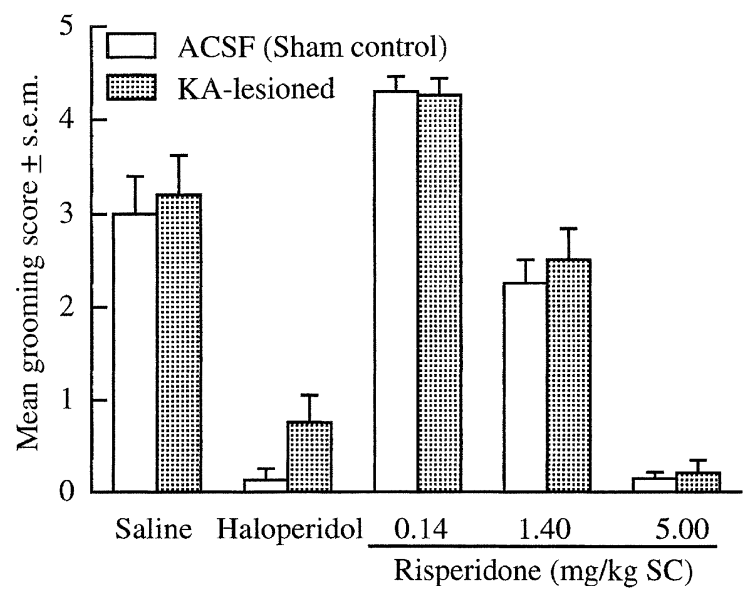

Figure 3. The effects of haloperidol and risperidone on grooming activity in KA-lesioned and control rats after amphetamine injection. Grooming activity was observed three times at 20-min intervals after amphetamine injection and each bar represents the average score for each group \pm SEM. Haloperidol and the two higher doses of risperidone significantly decreased post-amphetamine grooming scores. KA lesions did not significantly alter drug effects on grooming.

at a trend level. On the other hand, hippocampal damage did not alter the suppressive effects of a high dose of either risperidone or olanzapine. Overall, the results suggest that moderate doses of risperidone and perhaps olanzapine require hippocampal neurons to completely exert their suppressive effects on locomotor activity, but higher doses of each drug are able to reduce locomotor activity in a hippocampal-independent manner.

As noted in the introduction, suppression of spontaneous and amphetamine-elevated locomotor activity in animals has served as a preclinical predictor of antipsychotic drug efficacy. Most current antipsychotic drugs may differ in the degree to which they suppress spontaneous and amphetamine-elevated locomotor activity, but most importantly, all known antipsychotic drugs do suppress both forms of activity (Arnt 1995). The purpose of this study was not to compare the amount of locomotor suppression produced by haloperidol, risperidone, and olanzapine, since many previous studies have already addressed that issue (see Ogren 1996 for review). It is not our position that less overall locomotor suppression should be necessarily construed as less efficacious, i.e. that olanzapine or even clozapine (Bardgett et al. 1998) are less efficacious than haloperidol or risperidone because of the former drugs' more moderate effects on locomotion. Instead, the primary purpose of this study was to determine only if the locomotor suppressive effect of each individual drug could be altered by hippocampal damage. Based on the data from each lesion vs. control comparison, hippocampal neuronal loss was indeed found to alter locomotor responses to haloperidol, a moderate dose of risperidone, and to some extent, a moderate dose of olanzapine. Does a lesion-induced attenuation of an antipsychotic's ability to suppress locomotion signify that a hippocampal deficit undermines an antipsychotic drug's efficacy? That question cannot be answered with the present data, but will hopefully be addressed in future preclinical and clinical studies.

Why might an intact hippocampus be required for the locomotor suppressive effects of haloperidol, a moderate dose of risperidone, and to some extent, a moderate dose of olanzapine? Perhaps part of the locomotor suppressive effect of each drug involves directly modifying limbic-cortical function or indirectly altering brain regions which receive limbic-cortical input. Relatively little is known about if and how antipsychotic drugs directly affect neuronal activity in limbic-cortical regions, such as the hippocampus or amygdala. Acute administration of haloperidol, clozapine, or high doses of risperidone decreases glucose utilization in the hippocampus, suggesting that these drugs can directly alter limbic-cortical function (Colangelo et al. 1997; Huang et al. 1999). However, since all three drugs similarly reduce hippocampal glucose utilization, such findings do not explain why KA-lesioned animals have different responses to haloperidol versus clozapine (Bardgett et al. 1998) or high doses of risperidone.

It is possible that KA-induced disruption of limbiccortical output to two key forebrain regions, the nucleus accumbens and the prefrontal cortex, changes the acute suppressive effect of some antipsychotic drugs. Both the nucleus accumbens and prefrontal cortex have been implicated in the locomotor activating effects of amphetamine and the suppressive effects of antipsychotic drugs, and each area receives excitatory input from limbic-cortical neurons (Sesack and Pickel 1990; Brog et al. 1993; Carr and Sesack 1996). Antipsychotic drugs block dopamine receptors in these areas and KA lesions can increase $D_{2}$ receptor density in the nucleus accumbens (Bardgett et al. 1995). However, it is doubtful that a change in $D_{2}$ receptor density alters the drug response in lesioned animals, since all antipsychotic drugs antagonize dopamine $\mathrm{D}_{2}$ receptors, but not all drugs or all drug doses were affected by the lesion (Bardgett et al. 1998). Our findings suggest that there is a non-dopaminergic component, possibly serotonergic, noradrenergic (Arnt 1995; Darracq et al. 1998), or another, that makes a more substantial contribution to amphetamine-elevated locomotor activity in KA-lesioned animals. This nondopaminergic component may be more sensitive to clozapine and to higher doses of olanzapine and risperidone, given their affinity for other receptors, such as serotonin, noradrenaline, muscarinic, and histaminergic sites (Schotte et al. 1993; Bymaster et al. 1996).

The effects of antipsychotic drugs on post-amphetamine grooming behavior were also assessed in this study. The indices of grooming that were recorded are similar to measures of stereotypy used by other investiga- 


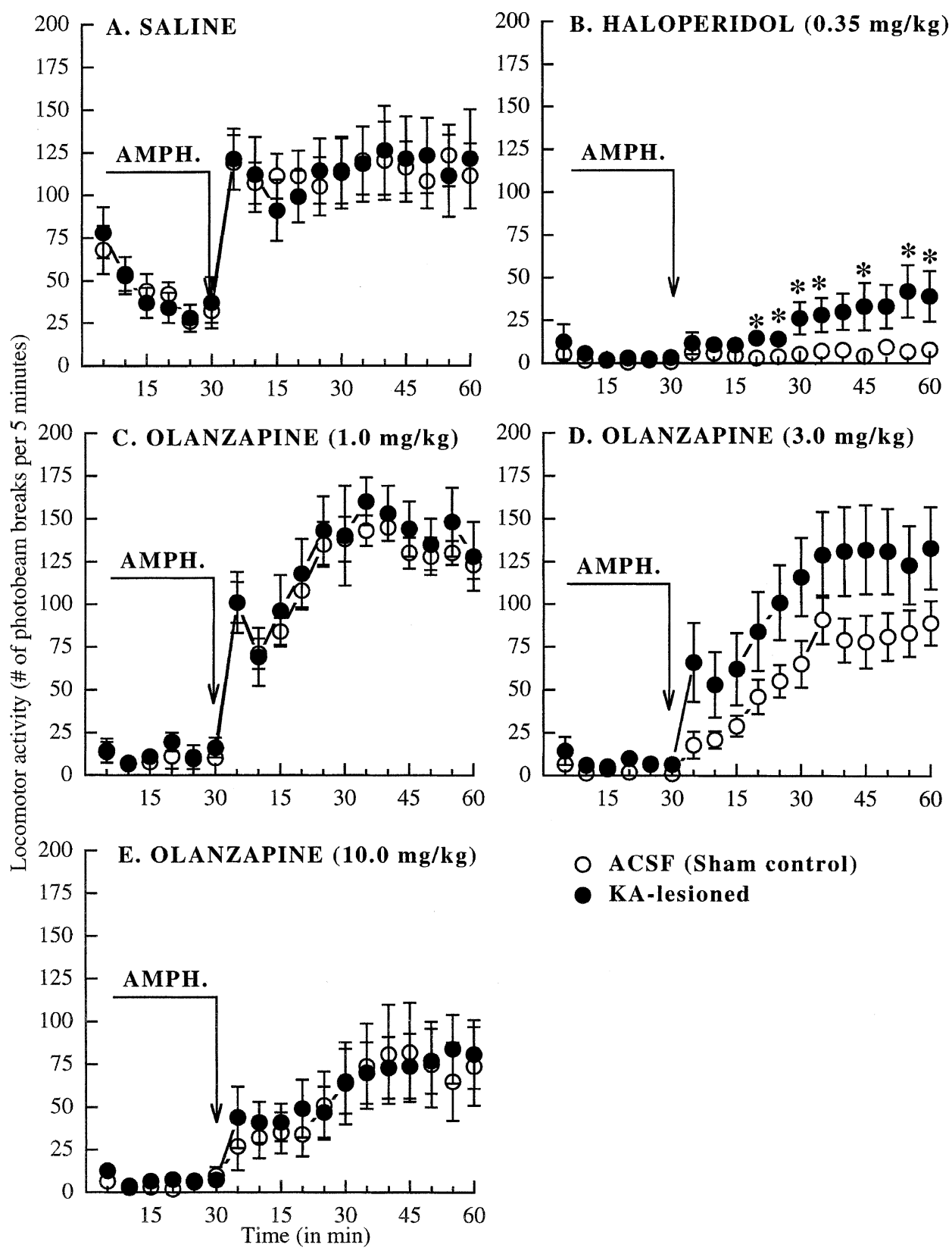

Figure 4. The effects of haloperidol and olanzapine on spontaneous and amphetamineelevated locomotor activity in KA-lesioned and control rats. The drug listed at the top of each graph was injected into each animal $30 \mathrm{~min}$ prior to the recording of locomotor activity. Each circle represents the mean $( \pm$ SEM) number of photobeam breaks recorded from each group during 5-min bins. Spontaneous locomotor activity was recorded for the first $30 \mathrm{~min}$. After this period, D-amphetamine $(1.5 \mathrm{mg} / \mathrm{kg}$, $\mathrm{SC}$ ) was injected into each animal, and locomotor activity was recorded for another $60 \mathrm{~min}$. Asterisks denote statistically significant differences between groups at individual time points $(p<.05$, Fisher's PLSD).

tors (Mittleman et al. 1991, 1993; Prosser et al. 1989). These behavioral measures were intended to serve as an indirect assay of amphetamine-induced nigrostriatal function in KA-lesioned and control animals (Mittleman et al. 1991, 1993) and have been used as a preclinical predictor of antipsychotic-induced motor side-effects (Ogren 1996). Lesions did not significantly alter the effects of risperidone or olanzapine on grooming behavior at any dose. While the lesions appeared to prevent the suppressive effects of haloperidol on grooming behavior, the effect of the lesion was not statistically significant. Taken together with the locomotor data, the findings suggest that limbic-cortical neurons play a greater role in locomotor responses to some antipsychotic drugs, and less of a role in the effects of the same drugs on non-ambulatory movements.
These data are consistent with the relatively greater input of limbic-cortical regions to locomotor centers in the brain, such as the nucleus accumbens, relative to areas that regulate non-ambulatory movements, such as the dorsal striatum (Sesack and Pickel 1991; Brog et al. 1993). Finally, it is notable that, independent of the lesion, the two higher doses of risperidone reduced post-amphetamine grooming behavior significantly, while the highest dose of olanzapine had no effect and the moderate dose actually increased grooming behavior. These data would suggest that olanzapine may have a lower propensity for producing extrapyramidal side effects relative to risperidone.

The KA lesion model was chosen for this study since there are several similarities between this model and the neuroanatomical and neurochemical changes asso- 


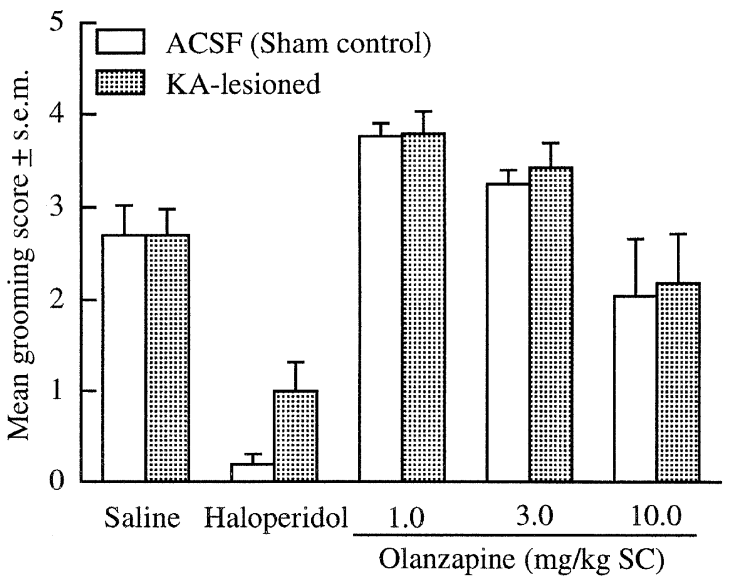

Figure 5. The effects of haloperidol and olanzapine on grooming activity in KA-lesioned and control rats after amphetamine injection. Grooming activity was observed three times at $20 \mathrm{~min}$ intervals after amphetamine injection and each bar represents the average score for each group \pm SEM. Haloperidol significantly decreased post-amphetamine grooming scores while the two lower doses of olanzapine significantly increased them. KA lesions did not significantly alter drug effects on grooming.

ciated with disorders treated with antipsychotic drugs, namely schizophrenia. As reviewed by Csernansky and Bardgett (1998), there have been reports of reduced hippocampal neuronal number, neuronal size, and hippocampal volume in schizophrenia, as well as reduced glutamate receptor expression. Kainic acid lesions produce similar effects in animals (Bardgett et al. 1995; Csernansky et al. 1998; M.E. Bardgett, unpublished data) without creating complete tissue loss in the hippocampus or other limbic-cortical structures. Furthermore, KA lesions elevate D2 receptor levels in the nucleus accumbens which may be analogous to the increased striatal D2 receptor density reported in some people with schizophrenia (Joyce et al. 1988; Wong et al. 1997). Finally, KA lesions elevate behavioral sensitivity to psychotomimetic drugs, such as amphetamine and N-methyl-D-aspartate (NMDA) antagonists (Bardgett et al. 1997a) and produce spatial working and contextual memory deficits (Roe et al. 1998; Yin et al. 2002) that resemble the cognitive impairments observed in schizophrenia (Park and Holzman 1992; Cohen et al. 1999).

The results from the present study suggest that the KA model may serve as a preclinical tool for understanding the role of hippocampal neurons in antipsychotic drug action. Future work in this area should consider lesion effects on antipsychotic-induced suppression of other forms of hyperlocomotion (i.e. NMDA antagonist-elevated activity). More importantly, continued research using KAlesioned animals should assess the effects of chronic antipsychotic treatment, which could provide more clinically relevant insights into limbic-cortical control of antipsychotic drug action. It is hoped that such research will ultimately improve our understanding of the role of hippocampal dysfunction in psychiatric disorders.

\section{ACKNOWLEDGMENTS}

This work was supported by a research grant from Eli Lilly and Co. to John Csernansky and by MH01109 to Mark Bardgett. We would like to thank Dr. Frank Bymaster for his valuable comments and Dr. Ray Richardson for his photographic assistance.

\section{REFERENCES}

Arnt J (1995): Differential effects of classical and newer antipsychotics on the hypermotility induced by two dose levels of D-amphetamine. Eur J Pharmacol 283:55-62

Bardgett ME, Jackson JL, Taylor GT, Csernansky JG (1995): Kainic acid decreases hippocampal neuronal number and increases dopamine receptor binding in the nucleus accumbens: An animal model of schizophrenia. Behav Brain Res 70:153-164

Bardgett ME, Csernansky JG (1996): Antipsychotic drug action after lesions to the hippocampus or prefrontal cortex. In Csernansky JG (ed), Handbook of Experimental Pharmacology: Antipsychotics, vol 120. Heidelberg, Springer-Verlag, pp 267-288

Bardgett ME, Jacobs PS, Jackson JL, Csernansky JG (1997a): Kainic acid lesions enhance locomotor responses to novelty, saline, amphetamine, and MK-801. Behav Brain Res $84: 47-55$

Bardgett ME, Salaris SL, Jackson JL, Harding J, Csernansky JG (1997b): The effects of kainic acid lesions on dopaminergic responses to haloperidol and clozapine. Psychopharmacology (Berl) 133:142-151

Bardgett ME, Jackson JL, Taylor BM, Csernansky JG (1998): The effects of kainic acid lesions on locomotor responses to haloperidol and clozapine. Psychopharmacology (Berl) 135:270-278

Bardgett ME, Henry JD (1999): Locomotor activity and accumbens fos expression driven by hippocampal stimulation requires D1 and D2 receptors. Neurosci 94:59-70

Brog JS, Salyapongse A, Deutch AY, Zahm DS (1993): The patterns of afferent innervation of the core and shell in the "accumbens" part of the rat ventral striatum: Immunohistochemical detection of retrogradely transported fluoro-gold. J Comp Neurol 338:255-278

Bymaster FP, Calligaro DO, Falcone JF, Marsh RD, Moore NA, Tye NC, Seeman P, Wong DT (1996): Radioreceptor binding profile of the atypical antipsychotic olanzapine. Neuropsychopharmacology 14:87-96

Carr DB, Sesack SR (1996): Hippocampal afferents to the rat prefrontal cortex: Synaptic targets and relation to dopamine terminals. J Comp Neurol 369:1-15

Cohen JD, Barch DM, Carter C, Servan-Schreiber D (1999): Context-processing deficits in schizophrenia: converg- 
ing evidence from three theoretically motivated cognitive tasks. J Abnorm Psychol 108:120-133

Colangelo V, Di Grezia R, Passarelli F, Musicco M, Ponteri FE, Orzi F (1997): Differential effects of acute administration of clozapine or haloperidol on local cerebral glucose utilization in the rat. Brain Res 768:273-278

Csernansky JG, Bardgett ME (1998): Limbic-cortical neuronal damage and the pathophysiology of schizophrenia. Schiz Bull 24:231-248

Csernansky JG, Csernansky CA, Kogelman L, Montgomery EM, Bardgett ME (1998): Progressive neurodegeneration after ICV kainic acid administration in rats: Implications for schizophrenia? Biol Psychiatry 44:1143-1150

Csernansky JG, Wang L, Joshi S, Miller JP, Gado M, Kido D, McKeel D, Morris JC, Miller MI (2000): Early DAT is distinguished from aging by high-dimensional mapping of the hippocampus. Dementia of the Alzheimer type. Neurology 55:1636-43

Darracq L, Blanc S, Glowinski J, Tassin J-P (1998): Importance of the noradrenaline-dopamine coupling in the locomotor activating effects of D-amphetamine. J Neurosci 18:2729-2739

Deutch AY (1996): Sites and mechanisms of action of antipsychotic drugs as revealed immediate early gene expression. In Csernansky JG (ed), Handbook of Experimental Pharmacology: Antipsychotics, vol 102. Heidelberg, SpringerVerlag, pp 117-162

Huang Y-H, Tsai S-J, Huang H-J, Sim C-B (1999): The effect of acute administration of risperidone on local cerebral glucose administration. Eur J Pharmacol 370:257-261

Joyce JN, Lexow N, Bird E, Winokur A (1988): Organization of dopamine D1 and D2 receptors in the human striatum: receptor autoradiographic studies in Huntington's disease and schizophrenia. Synapse 2:546-557

Marder SR (1999): An approach to treatment resistance in schizophrenia. Br J Psychiatry 37:19-22

McCarley RW, Wible CG, Frumin M, Hirayasu Y, Levitt JJ, Fischer IA, Shenton ME (1999): MRI anatomy of schizophrenia. Biol Psychiatry 45:1099-1119

Mittleman G, Jones GH, Robbins TW (1991): Sensitization of amphetamine-stereotypy reduces plasma corticosterone: Implications for stereotypy as a coping response. Behav Neur Biol 56:170-182

Mittleman G, LeDuc PA, Whishaw IQ (1993): The role of D1 and $\mathrm{D} 2$ receptors in the heightened locomotion induced by direct and indirect dopamine agonists in rats with hippocampal damage: An animal analogue of schizophrenia. Behav Brain Res 55:253-267.

Nadler JV, Perry BW, Cotman C (1978): Preferential vulnerability of hippocampus to intraventricular kainic acid. In McGeer EG, Olney JW, McGeer PL (eds), Kainic Acid as a Tool in Neurobiology, 1st ed. New York, Raven Press, pp 219-237

Ogren SO (1996): The behavioral pharmacology of typical and atypical antipsychotic drugs. In Csernansky JG (ed), Handbook of Experimental Pharmacology: Antipsychotics. Heidelberg, Springer-Verlag, pp 225-266

Park S, Holzman PS (1992): Schizophrenics show spatial working memory deficits. Arch Gen Psychiatry 49:975982

Paxinos G, Watson C (1986): The rat brain in stereotaxic coordinates. Sydney, Academic Press

Prosser ES, Pruthi R, Csernansky JG (1989): Differences in the time course of dopaminergic supersensitivity following chronic administration of haloperidol, molindone, or sulpiride. Psychopharmacology 99:109-116

Roe DL, Bardgett ME, Csernansky CA, Csernansky JG (1998): Induction of fos protein by antipsychotic drugs in rat brain following kainic acid-induced limbic-cortical neuronal loss. Psychopharmacology (Berl) 138:151-158

Schotte A, Janssen PFM, Megens AAHP, Leysen JE (1993): Occupancy of central neurotransmitter receptors by risperidone, clozapine and haloperidol, measured ex vivo by quantitative autoradiography. Brain Res 631:191-202

Sesack SR, Pickel VM (1990): In the rat nucleus accumbens, hippocampal and catecholaminergic terminals converge on spiny neurons and are in apposition to each other. Brain Res 527:266-279

Tollefson GD, Kuntz AJ (1999): Review of recent clinical studies with olanzapine. Br J Psychiatry 37:30-35

Yin H, Bardgett ME, Csernansky JG (2002): Kainic acid lesions disrupt fear-mediated memory processes. Neurobiol Learn Mem 77:389-401

Wong DF, Pearlson GD, Tune LE, Young LT, Meltzer CC, Dannals RF, Ravert HT, Reith J, Kuhar MJ, Gjedde A (1997): Quantification of neuroreceptors in the living human brain: IV. Effect of aging and elevations of D2like receptors in schizophrenia and bipolar illness. J Cereb Blood Flow Metab 17:331-342 